\section{SAÚDE E AMBIENTE}

V.8・N.3 2021 - Fluxo Contínuo

ISSN Digital: 2316-3798

ISSN Impresso: 2316-3313

DOI: 10.17564/2316-3798.2021v8n3p124-137

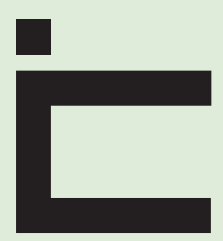

开N至医

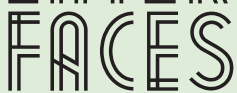

CIENTÍFICAS

\section{QUALIDADE DA ASSISTÊNCIA INTERVENCIONISTA PRESTADA PELO SERVIÇO DE ATENDIMENTO MÓVEL DE URGÊNCIA EM ARACAJU SERGIPE, BRASIL}

QUALITY OF INTERVENTIONIST CARE PROVIDED BY THE MOBILE EMERGENCY CARE SERVICE IN ARACAJU, SERGIPE, BRAZIL

CALIDAD DE LA ASISTENCIA INTERVENCIONISTA OFRECIDA POR EL SERVICIO DE ATENCIÓN MÓVIL DE URGENCIA EN ARACAJU, SERGIPE, BRASIL
Joathan Borges Ribeiro ${ }^{1}$

Josefa Jadiane dos Santos ${ }^{2}$ Hendyara Oliveira Carvalho Almeida ${ }^{3}$

Daniele Martins de Lima ${ }^{4}$ Ingrid Almeida de Melo $^{5}$

\section{RESUMO}

No Brasil, a baixa qualidade da assistência à saúde é evidenciada em grande parte dos serviços ofertados pelo Sistema Único de Saúde (SUS). Este estudo teve por objetivo avaliar a qualidade da assistência prestada pelo SAMU-192 na cidade de Aracaju/SE. Estudo transversal e descritivo, com abordagem quantitativa, realizado nas bases do SAMU-192 localizadas no município de Aracaju/SE com a aplicação do instrumento Avaliação da Qualidade da Assistência Pré-Hospitalar (AQ-APH), adaptado aos profissionais intervencionistas atuantes nesse serviço. Os resultados da pesquisa foram apresentados em três categorias: a avaliação dos indicadores referentes à estrutura que foi classificada "bom" pela maioria dos profissionais, aos processos que demonstrou maior parte dos aspectos julgados positivamente, com um grande percentual aos níveis "bom" ou "excelente" à classificação quanto à qualidade do serviço que apresentou-se de forma adequada uma vez que ao somar a pontuação concedida aos indicadores avaliados, atingiu-se 13 pontos, dentre 17 possíveis, ultrapassando, então, a referência estipulada $(\geq 10)$. 0 instrumento AQ-APH contribui de forma significativa para a identificação das deficiências que interferem na qualidade do serviço e o SAMU-192 no município de Aracaju, atende de forma satisfatória à população, sendo avaliado como adequado com uma pontuação de 13 pontos no total correspondente a 5 pontos da estrutura e 8 dos processos.

\section{PALAVRAS-CHAVE}

Serviços Médicos de Emergência. Avaliação em Saúde. Qualidade da Assistência à Saúde. 


\section{ABSTRACT}

In Brazil, the low quality of health care is evidenced in most of the services offered by the Health Unic System (SUS). Thus, the research had the general objective of evaluating the quality of the assistance provided by SAMU-192 in the city of Aracaju / SE. Cross-sectional and descriptive study, with a quantitative approach, carried out on the bases of SAMU-192 located in the municipality of Aracaju / SE with the application of the instrument Assessment of the Quality of Pre-Hospital Care (AQ-APH), adapted, to the active interventionist professionals in that service. The results of the research were presented in three categories: the evaluation of the indicators referring to the structure that was classified as "good" by most professionals, to the processes that demonstrated most of the aspects judged positively, with a large percentage at the "good" levels or "excellent and the classification as to the quality of the service, which was presented adequately, since adding the score given to the evaluated indicators, it reached 13 points, among 17 possible, and then exceeding the stipulated reference $(\geq 10)$. the evaluation of the indicators referring to the structure, processes and classification regarding the quality of service as a result of these components, with the structure having a score equal to 5 and the processes equal to 8 , being in agreement with the adequacy parameters, attribution of 1 point to each indicator considered "good" or "excellent" in the main, qualitatively characterizing them as adequate. The AQ-APH instrument contributes significantly to the identification of the deficiencies that interfere with the quality of the service and the SAMU-192 in the municipality of Aracaju, satisfactorily serves the population, being assessed as adequate with a score of 13 points in the total corresponding to 5 points of the structure and 8 of the processes.

\section{KEYWORDS}

Emergency medical services. Health evaluation. Quality of health care.

\section{RESUMEN}

En Brasil, la baja calidad de la atención de salud se evidencia en la mayoría de los servicios que ofrece el Sistema Único de Salud (SUS). Así, la investigación tuvo como objetivo general evaluar la calidad de la asistencia brindada por SAMU-192 en la ciudad de Aracaju/SE. Estudio descriptivo, transversal, con abordaje cuantitativo, realizado sobre las bases del SAMU-192 ubicado en el municipio de Aracaju/SE con la aplicación del instrumento Evaluación de la Calidad de la Atención Prehospitalaria (AQ-APH), adaptada, a los profesionales intervencionistas activos en ese servicio. Los resultados de la investigación se presentaron en tres categorías: la evaluación de los indicadores referentes a la estructura que fue calificada como "buena” por la mayoría de los profesionales, a los procesos que 
demostraron la mayoría de los aspectos juzgados positivamente, con un gran los niveles de "bueno" o "excelente" y la clasificación en cuanto a la calidad del servicio, que se presentó de manera adecuada, ya que sumando el puntaje otorgado a los indicadores evaluados, llegó a 13 puntos, entre 17 posibles, para luego superar la referencia estipulada $(\geq 10)$. la evaluación de los indicadores referentes a la estructura, procesos y clasificación en cuanto a la calidad de servicio como resultado de estos componentes, teniendo la estructura un puntaje igual a 5 y los procesos igual a 8 , estando de acuerdo con los parámetros de adecuación, mediante la atribución de 1 punto a cada indicador considerado "bueno" o "excelente" en su mayor parte, caracterizándolos cualitativamente como adecuados. El instrumento AQ-APH contribuye significativamente a la identificación de las deficiencias que interfieren con la calidad del servicio y el SAMU-192 en el municipio de Aracaju, atiende satisfactoriamente a la población, siendo evaluado como adecuado con un puntaje de 13 puntos en el total correspondiente a 5 puntos de la estructura y 8 de los procesos.

\section{PALABRAS CLAVE}

Servicios médicos de urgencia; Evaluación en Salud; Calidad de la Atención de Salud.

\section{INTRODUÇ̄̃̃O}

No Brasil, a baixa qualidade da assistência à saúde é evidenciada em grande parte dos serviços ofertados pelo Sistema Único de Saúde (SUS), sendo este problema de origem multifatorial, contemplando desde a infraestrutura até a metodologia de trabalho utilizada. Atualmente, a aplicação de ferramentas avaliativas como método de qualificação da assistência é amplamente utilizada nas instituições de saúde, no entanto, apesar da sua importante contribuição na melhoria dos serviços, no Atendimento Pré-Hospitalar (APH) móvel de urgência, a utilização desses instrumentos ainda é escassa (ORTIGA et al., 2016).

$\mathrm{O} A \mathrm{PH}$ consiste em todo atendimento à saúde prestado no ambiente extra-hospitalar que visa à estabilização do quadro clínico e a prevenção de complicações futuras do paciente, inclusive a morte, até a chegada à instituição de saúde de referência onde será realizado o tratamento definitivo. No contexto brasileiro, esse tipo de atendimento é classificado em fixo e móvel, sendo este desenvolvido pelo Serviço de Atendimento Móvel de Urgência (SAMU-192) que atende às necessidades de saúde da população, sejam eles de etiologia clínica, cirúrgica, traumática e até mesmo psiquiátrica (O’DWYER et al., 2017).

No estado de Sergipe, a Central de Regulação de Urgências (CRU) registrou no ano de 2016 um total de mais de 103 mil ligações ao SAMU-192, em que 51\% resultaram em atendimento, sendo 20,5\% por Unidades de Suporte Avançado (USA), 76,64\% por Unidades de Suporte Básico (USB) e 2,83\% por motolância. Em 2017, foram recebidas pelo serviço 224.683 chamadas, mais que o dobro dos telefonemas ocorridos no ano anterior, entretanto, quanto aos atendimentos gerados apenas $4,83 \%$ destas resultaram em assistência por USA e 15,30\% por USB (SERGIPE, 2018). 
Destarte, devido à significância deste serviço para a população é necessária a avaliação da qualidade deste que consiste em uma ferramenta de grande relevância para o monitoramento contínuo, detecção e correção dos desvios dos padrões de maneira precoce. Para facilitar este processo, são utilizadas estratégias, a exemplo dos indicadores, de forma a subsidiar a gestão em saúde, uma vez que possui grande importância nos serviços de urgência, pois o nível de assistência prestada exige uma resposta rápida às necessidades de saúde por meio de um trabalho ágil e dinâmico (OLIVEIRA et al., 2018).

No entanto, no serviço móvel de urgência, esses mecanismos ainda são pouco utilizados, havendo apenas um único instrumento validado para essa finalidade, denominado Avaliação da Qualidade da Assistência Pré-Hospitalar (AQ-APH). Este foi criado com base na Tríade Donabediana que consiste em um modelo conceitual destinado à exploração e avaliação da qualidade dos cuidados prestados pelos serviços de saúde por meio da extração de informações em três dimensões: estrutura, processo e resultado. O AQ-APH é composto por 17 indicadores que contempla duas das três vertentes da tríade: a estrutura que analisa os recursos humanos, físicos, materiais e financeiros e o processo que avalia as atividades que envolvem os profissionais e usuários (DANTAS et al., 2015).

Diante do exposto, justifica-se o presente estudo pela escassez de pesquisas que abordem a temática em questão no Brasil e pela necessidade de avaliar a qualidade da assistência prestada pelo SAMU-192 na cidade de Aracaju/SE com a finalidade de identificar os principais nós críticos que comprometem a qualidade do serviço para elaboração do diagnóstico situacional e consequentemente, auxiliar os gestores no desenvolvimento e implementação de ações que visem à melhoria do quadro atual, visto que ele consiste em um importante componente da Rede Estadual de Saúde.

Logo, esta pesquisa teve como objetivo avaliar a qualidade da assistência prestada pelo SAMU-192 na cidade de Aracaju/SE por meio da análise da estrutura e dos processos que compõem o serviço.

\section{METODOLOGIA}

A presente pesquisa é do tipo transversal e descritiva, com abordagem quantitativa. Esta foi realizada nas bases do SAMU-192 localizadas no município de Aracaju/SE no mês de outubro de 2018. Elas dispõem de três USA e oito USB, além de quatro motolâncias e uma população de funcionários assistenciais intervencionistas composta por 224 indivíduos: 105 técnicos e auxiliares de enfermagem, 77 condutores, 21 enfermeiros e 21 médicos.

Para o plano amostral, foi adotado o teste Qui-Quadrado de Pearson. Assumindo um tamanho de efeito médio ( $w=0,3)$, um poder de $80 \%$ ), uma significância de $5 \% 0$ e 3 graus de liberdade (v). Com isso, o tamanho de amostra ideal foi de 122 participantes, seguindo a equação proposta por Cohen (1992).

Além disso, foi somado a este, $10 \%$ da amostra ideal como percentual de segurança para absenteísmo e possíveis desistências após preenchimento do instrumento e do Termo de Consentimento Livre e Esclarecido (TCLE). Dessa forma, a amostra final do estudo foi constituída por 135 profissionais.

Foram incluídos na pesquisa os profissionais que prestam uma assistência intervencionista à população, alocados em um dos veículos de urgência do SAMU-192 Sergipe na cidade de Aracaju, com 
idade igual ou superior a dezoito anos. Foram excluídos aqueles que estavam de licença, sendo ela de quaisquer naturezas, férias, assim como os que não aceitaram o TCLE.

A coleta de dados foi realizada pelos discentes da pesquisa após a aprovação do Comitê de Ética e Pesquisa (CEP) da Universidade Tiradentes por meio do parecer de número 2.957.177. Os profissionais foram abordados nas referidas bases durante o plantão em que estiveram escalados e entrevistados após a assinatura do TCLE em duas vias. Para tal, foi utilizado o instrumento AQ-APH adaptado, sendo este construído e validado por Dantas e colaboradores (2015).

0 instrumento em questão é destinado especificamente para o serviço móvel de urgência e avalia a qualidade da assistência prestada por meio da análise da estrutura e dos processos que compõem o mesmo. Tal ferramenta possui 17 indicadores: estado de conservação das ambulâncias, estrutura física, conforto da ambulância, recursos materiais, segurança do usuário/profissional, educação permanente, segurança demonstrada pela equipe, acesso, acolhimento, humanização, tempo resposta, privacidade ao usuário, orientações sobre o atendimento, relacionamento entre o profissional e usuário, oportunidade de o usuário realizar reclamações e articulação multiprofissional (DANTAS et al., 2015).

A análise dos dados seguiu, parcialmente, a metodologia proposta por Dantas e colaboradores (2015). As variáveis foram caracterizadas em duas formas para realização estatística, inicialmente as respostas para cada indicador foram apresentadas na conformação de escala do tipo Likert, composta por cinco níveis no instrumento em questão: péssimo; ruim; regular; bom; e excelente. Posteriormente, em meio à análise dos dados correspondente à avaliação da qualidade da assistência prestada, segundo os profissionais, as respostas: péssimo; ruim; e regular foram enquadradas na variável qualitativa - "inadequado", enquanto bom e excelente, como "adequado".

As variáveis, correspondentes aos indicadores de estrutura e processo, foram classificadas em categorias quantitativas. Aos itens considerados como adequados atribuiu-se um ponto a cada um deles, obtendo um total entre 0 e 17 pontos, visto que o instrumento contém 8 itens relacionados à estrutura e 9 itens ao processo. Nessa perspectiva, julgou-se "adequado" o aspecto que obteve pontuação acima da metade de seu total, sendo estrutura $>4$ pontos e processo $\geq 5$ pontos e "inadequado" aqueles que não se enquadrarem nestes parâmetros. Ao analisar as duas dimensões juntas, foi considerado que o serviço está "adequado" para os escores $\geq 10$ pontos e "inadequado" os escores inferiores a esse valor.

Os dados coletados foram digitados em planilha do programa Microsoft Excel 2016 versão 1807 e analisados estatisticamente com o auxílio do Software Stata versão 15. A apresentação das variáveis numéricas ocorreu por meio de suas medidas de tendência central e dispersão (média e intervalo de confiança, mediana, mínimo, máximo, entre outros) e as variáveis categóricas em frequências absolutas e relativas - representadas em percentual, sendo estas associadas por meio do teste Qui-quadrado de Pearson. 0 nível de significância adotado foi de $5 \%$.

0 presente estudo obedeceu aos princípios éticos que regem as pesquisas que envolvem seres-humanos de acordo com a Resolução no 466/12 e 510/16 do Conselho Nacional de Saúde (CNS). Além disso, também foi utilizada a Resolução $n^{0}$ 580/18 que dispõem sobre os parâmetros éticos a serem considerados em pesquisas realizadas com seres humanos em instituições que integram o SUS. 


\section{RESULTADOS}

Os resultados foram compilados por área de avaliação sendo apresentados os aspectos estruturais, relacionados aos processos e a classificação da qualidade do serviço em questão.

Tabela 1 - Distribuição de frequência dos aspectos sobre a estrutura para avaliação da qualidade da assistência do SAMU - 192 na cidade de Aracaju, SE, no ano de 2018

\begin{tabular}{|c|c|c|c|c|c|}
\hline Indicadores & $\begin{array}{l}\text { Péssimo } \\
\text { n (\%) }\end{array}$ & $\begin{array}{l}\text { Ruim } \\
\text { n (\%) }\end{array}$ & $\begin{array}{l}\text { Regular } \\
\text { n (\%) }\end{array}$ & $\begin{array}{l}\text { Bom } \\
\text { n (\%) }\end{array}$ & $\begin{array}{l}\text { Excelente } \\
\mathbf{n}(\%)\end{array}$ \\
\hline $\begin{array}{l}\text { O estado de conservação das ambu- } \\
\text { lâncias é? }\end{array}$ & $14(9,8)$ & $13(9,1)$ & $38(26,6)$ & $47(32,9)$ & $31(21,7)$ \\
\hline A estrutura física nesse serviço é? & $19(13,3)$ & $29(20,3)$ & $66(46,2)$ & $26(18,2)$ & $3(2,1)$ \\
\hline O conforto dentro da ambulância é? & $10(7,0)$ & $17(11,9)$ & $40(28,0)$ & $57(39,9)$ & $19(13,3)$ \\
\hline $\begin{array}{c}\text { A oferta de segurança ao usuário } \\
\text { dentro da ambulância é? }\end{array}$ & $7(4,9)$ & $22(15,4)$ & $45(31,5)$ & $48(33,6)$ & $21(14,7)$ \\
\hline $\begin{array}{l}\text { A disponibilidade de materiais para } \\
\text { seu entendimento foi? }\end{array}$ & $1(0,7)$ & $7(4,9)$ & $41(28,7)$ & $73(51,0)$ & $21(14,7)$ \\
\hline $\begin{array}{c}\text { A segurança demonstrada pela equi- } \\
\text { pe durante o atendimento geralmen- } \\
\text { te é? }\end{array}$ & $3(2,1)$ & $6(4,2)$ & $17(11,9)$ & $72(50,3)$ & $45(31,5)$ \\
\hline $\begin{array}{c}\text { A segurança da equipe em relação } \\
\text { aos pontos de apoio (PAs) oferecidos } \\
\text { por esses serviços são? }\end{array}$ & $28(19,6)$ & $28(19,6)$ & $56(39,2)$ & $24(16,8)$ & $7(4,9)$ \\
\hline $\begin{array}{c}\text { A educação permanente nesses } \\
\text { serviços é? }\end{array}$ & $9(6,3)$ & $17(11,9)$ & $43(30,1)$ & $51(35,7)$ & $23(16,1)$ \\
\hline
\end{tabular}

Legenda: $\mathrm{n}$ - Frequência Absoluta; \% - Frequência Relativa

Fonte: Dados da pesquisa.

A Tabela 1 demonstra os aspectos estruturais do SAMU-192 na cidade de Aracaju no ano de 2018, com base na opinião da maioria dos profissionais intervencionistas que participaram da pesquisa, o serviço é classificado como bom, no que se refere ao estado de conservação das ambulâncias, ao conforto dentro desse veículo, a oferta de segurança ao usuário dentro dele, a disponibilidade de materiais, a segurança demonstrada pela equipe durante dos atendimentos e a educação permanente. Entretanto, a estrutura física e a segurança da equipe nos pontos de apoio foram classificadas como regular pela maior parte dos entrevistados. A seguir é apresentada Tabela 2 com a avaliação de aspectos relacionados aos processos. 
Tabela 2 - Distribuição de frequência dos aspectos sobre os processos para avaliação da qualidade da assistência do SAMU-192 na cidade de Aracaju, SE, no ano de 2018

\begin{tabular}{|c|c|c|c|c|c|}
\hline Indicadores & $\begin{array}{l}\text { Péssimo } \\
\text { n (\%) }\end{array}$ & $\begin{array}{l}\text { Ruim } \\
\text { n (\%) }\end{array}$ & $\begin{array}{l}\text { Regular } \\
\text { n (\%) }\end{array}$ & $\begin{array}{l}\text { Bom } \\
n(\%)\end{array}$ & $\begin{array}{c}\text { Excelente } \\
\text { n (\%) }\end{array}$ \\
\hline O acesso ao serviço pelo número 192 é? & $4(2,8)$ & $6(4,2)$ & $48(33,6)$ & $68(47,6)$ & $17(11,9)$ \\
\hline $\begin{array}{l}\text { O tempo de chegada da ambulância ao } \\
\text { local de chamado é? }\end{array}$ & $1(0,7)$ & $16(11,2)$ & $45(31,5)$ & $65(45,5)$ & $16(11,2)$ \\
\hline $\begin{array}{l}\text { O acolhimento dispensado nesse serviço } \\
\text { pelos profissionais aos usuários é? }\end{array}$ & $1(0,7)$ & $9(6,3)$ & $29(20,3)$ & $58(40,6)$ & $46(32,2)$ \\
\hline $\begin{array}{l}\text { Durante o atendimento, o respeito à pri- } \\
\text { vacidade dos usuários nesse serviço é? }\end{array}$ & $2(1,4)$ & $5(3,5)$ & $28(19,6)$ & $55(38,5)$ & $53(37,1)$ \\
\hline $\begin{array}{c}\text { A humanização durante a assistência } \\
\text { prestada pela equipe é? }\end{array}$ & $2(1,4)$ & $4(2,8)$ & $21(14,7)$ & $62(43,4)$ & $54(37,8)$ \\
\hline $\begin{array}{l}\text { As orientações sobre os procedimentos } \\
\text { realizados e estado de saúde, forneci- } \\
\text { das pela equipe do SAMU-192 SE, aos } \\
\text { usuários nesse serviço são? }\end{array}$ & $1(0,7)$ & $7(4,9)$ & $38(26,6)$ & $65(45,5)$ & $32(22,4)$ \\
\hline $\begin{array}{l}\text { O relacionamento entre os profissionais } \\
\text { e os usuários nesse serviço é? }\end{array}$ & $1(0,7)$ & $10(7,7)$ & $38(26,6)$ & $74(51,7)$ & $20(14,0)$ \\
\hline $\begin{array}{l}\text { A oportunidade dada aos usuários de } \\
\text { fazer reclamações nesse serviço é? }\end{array}$ & $5(3,5)$ & $23(16,1)$ & $63(44,1)$ & $39(27,3)$ & $13(9,1)$ \\
\hline $\begin{array}{l}\text { A articulação do trabalho multiprofissio- } \\
\text { nal nesse serviço é? }\end{array}$ & $7(4,9)$ & $14(9,8)$ & $45(31,5)$ & $54(37,8)$ & $23(16,1)$ \\
\hline
\end{tabular}

Legenda: $\mathrm{n}$ - Frequência Absoluta; \% - Frequência Relativa

Fonte: Dados da pesquisa

Os indicadores de processo, apresentaram a maior parte dos aspectos julgados positivamente, com um grande percentual, aos níveis "bom" ou "excelente", a exemplo do acesso ao serviço pelo número 192 e o tempo de chegada da ambulância ao local chamado.

Índices expressivos foram observados nos resultados obtidos nos itens de acolhimento dispensado pelos profissionais aos usuários, o respeito à privacidade dos mesmos durante o atendimento e a humanização expressa pela equipe no transcorrer da intervenção. Vale ressaltar a frequência relativa de adequação obtida pelos indicadores que avaliaram as orientações fornecidas aos pacientes sobre os procedimentos realizados e estado de saúde pela equipe do SAMU em questão e o relacionamento entre profissionais e usuários nesse serviço que se associam.

A articulação do trabalho multiprofissional no SAMU-192 da cidade de Aracaju foi avaliada como adequada pela maioria dos profissionais participantes da pesquisa. Entretanto, a oportunidade dada 
aos usuários de fazer reclamações no serviço em questão foi considerada inadequada visto que a maioria dos profissionais participantes classificaram esse fator como péssimo, ruim ou regular.

A Tabela 3 apresenta o desfecho da avaliação do SAMU-192 da cidade de Aracaju como resultado da classificação das variáveis de estrutura e de processos. Este foi considerado "bom" ou "excelente" majoritariamente, caracterizando-os qualitativamente como adequado visto que sua pontuação total está acima do valor de referência estipulado. Quanto à estrutura, obteve-se um total que está em concordância com os parâmetros de adequação.

Tabela 3 - Classificação da qualidade do SAMU-192 na cidade de Aracaju, SE, como resultado das variáveis de estrutura, processos e serviços, no ano de 2018

\begin{tabular}{cccc}
\hline Variáveis & Pontuação & Valor de Referência & Avaliação \\
\hline Estrutura & 5 & $>4$ & Adequado \\
Processos & 8 & $\geq 5$ & Adequado \\
Serviços & 13 & $\geq 10$ & Adequado \\
\hline
\end{tabular}

Fonte: Dados da pesquisa

Destarte, o serviço em questão apresenta-se de forma adequada uma vez que ao somar a pontuação concedida aos indicadores avaliados, ultrapassou-se então a referência estipulada.

\section{DISCUSSÃO}

A qualidade em saúde pode ser avaliada por meio da observação e análise dos recursos humanos, físicos e financeiros disponíveis, associada à metodologia de desenvolvimento do processo de trabalho e dos resultados obtidos com o serviço ofertado, visto que são elementos que atuam de forma articulada (MELO et al., 2014).

O Serviço de Atendimento Móvel de Urgência é formado por uma totalidade de fatores que interferem na prestação de cuidados em uma instituição de saúde, incluindo os seus elementos estáveis, como: recursos físicos, humanos, financeiros, dentre outros. A avaliação da estrutura fornece poucas informações a respeito da qualidade da atenção prestada em relação aos demais elementos, entretanto, fundamenta os mesmos visto que problemas identificados neste aspecto ocasionam déficits nos processos e, consequentemente, nos resultados (DANTAS, 2014).

Nos resultados obtidos, nota-se que o estado de conservação, o conforto e a oferta de segurança ao usuário dentro da viatura apresentam percentual semelhantes, visto que, segundo relato dos profissionais entrevistados, a maioria desses veículos foram trocados recentemente e atendem satisfatoriamente às necessidades dos profissionais e usuários quanto aos aspectos questionados, contribuindo dessa forma para prestação de uma assistência qualificada e segura. 
A disponibilidade de materiais foi o item com maior percentual de avaliação positiva, pois, segundo a maioria dos entrevistados, poucas vezes aconteceu a ausência desses recursos essenciais para o atendimento e na ocorrência dessas situações, foram solucionadas rapidamente. Corroborando essa informação, Mata e colaboradores (2018) citam em seu estudo que objetivou identificar os entraves no APH do SAMU, em uma cidade do interior do Estado do Piauí, na percepção dos enfermeiros, a qualidade do atendimento prestado está diretamente ligada à disponibilidade e qualidade dos materiais, e para tal, além do adequado gerenciamento de enfermagem, é necessário que haja um controle e avaliação dos mesmos por parte de toda equipe multiprofissional.

No entanto, houve uma discrepância significativa de opiniões quanto à segurança demonstrada pela equipe na assistência e a qualidade da educação permanente, fato este preocupante, uma vez que se constitui como uma importante ferramenta de aprimoramento e atualização dos conhecimentos dos profissionais para que atuem de forma adequada e demonstrem segurança durante o atendimento. Segundo Coelho e colaboradores (2013), a educação permanente permite a aproximação entre a rotina do profissional e as necessidades de saúde dos usuários, de modo a desenvolver nos elementos envolvidos um raciocínio crítico frente as mais diversas situações, por meio da interação e da aquisição e aprimoramento dos conhecimentos.

Quanto à estrutura física e a segurança da equipe nos pontos de apoio, classificadas como regular, grande parte dos entrevistados relatou que essa conformação física do local não contribui para segurança, pois há uma deficiência no controle de acesso das pessoas ao estabelecimento. De acordo com a versão 2.0 do ano de 2018 do Programa Arquitetônico Mínimo para Base Descentralizada SAMU-192, é necessário que a mesma disponha de instalações que proporcionem abrigo, alimentação, conforto das equipes e estacionamento das ambulâncias, além de garantir segurança dos profissionais e dos materiais e equipamentos que se encontram no local (BRASIL, 2017).

Os processos constituem-se nos elementos que compõem as práticas de prestação dos cuidados em saúde vinculados com os elementos que interferem na relação profissional/usuário e engloba os cuidados preventivos, o diagnóstico, o tratamento e a educação realizada ao paciente. Dessa forma, esse item abrange a maneira pela qual os cuidados são prestados (SILVA et al., 2015).

O desfecho positivo observado nos quesitos de acesso ao serviço pelo número 192 e o tempo de chegada da ambulância ao local chamado foi observado também em estudo realizado na cidade de Maringá (PR) a qual obteve $77,6 \%$ e $86,2 \%$ respectivamente, quanto aos dois indicadores citados anteriormente classificados como "bom” ou “excelente" (GARÇON; PUPULIM, 2017).

Todavia, mesmo com a superioridade na satisfação expressa pelos profissionais entrevistados quanto ao acesso da população pelo número 192, 40,5\% discordaram de tal item, situação que demonstra uma contraposição do princípio fundamental do SUS, a universalidade. Além disso, 42,9\% demonstraram insatisfação no que se refere ao tempo de chegada da viatura em cena, fato esse motivado pelos diversos interferentes nesse indicador, a exemplo da localização das ambulâncias, da recepção dos chamados, do número de equipes atuantes e dos protocolos destinados à classificação das ocorrências (O’DWYER et al., 2017).

No entanto, os percentuais mais expressivos no que tange aos processos, foram quesitos considerados adequados que se voltam a fatores resultantes da humanização na assistência presta- 
da como o acolhimento dispensado pelos profissionais aos usuários, o respeito à privacidade dos mesmos durante o atendimento e a própria humanização expressa pela equipe no transcorrer da intervenção. Dessa forma, reconhecer as necessidades de saúde expressas de maneira singular pelos indivíduos por meio do devido acolhimento e promover uma ambiência que preconize a privacidade do usuário propiciam mudanças no processo de trabalho, responsáveis pelo fortalecimento na execução do humanizar (VITURI et al., 2013).

Além disso, a conformidade identificada quanto às orientações fornecidas aos pacientes sobre os procedimentos realizados, estado de saúde pela equipe do SAMU em questão, o relacionamento entre profissionais e usuários nesse serviço reafirmam a humanização da equipe assistencial e constituem-se como ferramentas para o envolvimento do usuário na promoção de sua segurança em meio ao processo assistencial, partindo da premissa que todo cidadão possui o direito da informação acerca do seu estado de saúde (ALVES et al., 2013).

Apesar do percentual favorável observado no tópico de articulação do trabalho multiprofissional no SAMU-192 da cidade de Aracaju, os níveis mais pontuados foram o "regular" (31,5\%) e o "bom" $(37,8 \%)$, sinalizando a necessidade de melhoria no relacionamento entre os funcionários deste serviço. Tal afirmação ocorre em conformidade à melhoria da comunicação entre os profissionais da saúde enquanto meta internacional para segurança do paciente e mecanismo promotor de compromisso na prestação de cuidados necessários às vítimas de agravos assistidas por eles (SILVA et al., 2014).

Grande enfoque tem-se dado à experiência do paciente em serviços de saúde, ponto que apresentou resultado desfavorável nesta pesquisa, avaliado por meio da oportunidade dada aos usuários de fazer reclamações no serviço em questão. Esta informação impossibilita a inserção desses indivíduos nos mecanismos de produção e gestão do cuidado e processos de trabalho, contrapondo-se ao princípio da transversalidade na prática da humanização por não viabilizar uma saúde de forma mais corresponsável por meio da valorização da experiência daqueles que foram assistidos pelo serviço (O'DWYER; MATTOS, 2013).

A qualidade da assistência prestada pelo SAMU-192 depende da metodologia de trabalho e dos recursos disponíveis para o seu desenvolvimento, além das competências e habilidades de toda a equipe de saúde com o objetivo principal de possibilitar que o paciente chegue com vida ao hospital de referência e com o mínimo de complicações possíveis. No entanto, para garantia de uma assistência adequada é necessário que este serviço seja avaliado em todos os seus aspectos, com o intuito de identificar as não conformidades e propor medidas corretivas para solucioná-las (DANTAS, 2014).

Por fim, o resultado de adequação observado aos aspectos de estrutura, processos e de todo o serviço avaliado opõe-se ao desfecho identificado em um estudo realizado com a aplicação do AQ-APH entre 179 profissionais do SAMU-192 do Rio Grande do Norte (RN), no qual foram considerados inadequados a avaliação da estrutura e do serviço em sua totalidade, por não atingirem o parâmetro estabelecido para uma avaliação qualitativa favorável (DANTAS, 2014).

Situação semelhante foi apontada na pesquisa realizada no SAMU-192 de Santa Catarina (SC) nos anos de 2013 e 2014, onde se identificou a inadequação deste serviço. Ele foi avaliado quanto a atenção à urgência por meio da infraestrutura, apoio diagnóstico e terapêutico e adequação desse 
fator, sendo concluído que havia a necessidade de maior atenção e investimento nesse serviço de APH do Estado, objetivando a eficácia deste enquanto ordenador da Rede de Urgência e Emergência (RUE) local (ORTIGA et al., 2016).

No entanto, apesar do SAMU-192 do município de Aracaju/SE apresentar adequação dos seus serviços, conforme avaliação do instrumento AQ-APH, cabe frisar que alguns itens foram julgados como deficientes. Dessa forma, há a necessidade de intervenção neles como medida de aprimoramento constante do serviço por meio de avaliações de rotina no funcionamento deste, visando a detecção precoce de interferentes que comprometam sua eficácia, visto que ele desempenha um papel fundamental na minimização de riscos e prevenções de agravos em situações de urgência e emergência.

\section{CONCLUSÃO}

Diante do exposto, percebe-se que o instrumento AQ-APH contribui de forma significativa para a identificação das deficiências que interferem na qualidade do serviço e para nortear os gestores na elaboração de estratégias que permitam a realização das adequações necessárias, tanto na estrutura quanto nos processos que compõem o mesmo, entretanto, tal instrumento apresenta algumas limitações, tais como: a não especificação dos problemas, sendo eles verificados apenas pelo relato verbal dos profissionais durante a entrevista, não havendo possibilidade de registrá-los.

Vale ressaltar, também, que a escassez de referencial teórico sobre o assunto abordado dificultou o desenvolvimento do presente estudo. Dessa forma, propões que mais estudos sejam realizados sobre a temática em questão para evidenciar a importância da estrutura e dos processos de trabalho que compõem tal Serviço de Saúde, tanto para Instituições e profissionais, como para os usuários, de modo a difundir o uso desse instrumento como ferramenta de inadequações e consequente melhora da qualidade da assistência ofertada, pode-se concluir que o serviço do SAMU-192 no município de Aracaju, atende de forma satisfatória à população, sendo avaliado como adequado com uma pontuação de 13 pontos no total correspondente a cinco pontos da estrutura e oito dos processos.

Contudo, para o alcance da excelência do serviço é necessário que as condições deficientes sejam verificadas criteriosamente pela gestão dele por meio da análise do relatório final da pesquisa, fornecido com uma síntese dos resultados obtidos pelos pesquisadores, para a elaboração do plano de melhorias pelos gestores, de acordo com a realidade do local.

\section{REFERÊNCIAS}

ALVES, M. et al. Particularidades do trabalho do enfermeiro no serviço de atendimento móvel de urgência de belo horizonte. Texto Contexto Enferm, v. 22, n. 1, p. 208-215, 2013. 
BRASIL. Ministério da Saúde. Portaria de Consolidação nº 3 de 03 de outubro de 2017. Consolidação das normas sobre as redes do Sistema Único de Saúde. Diário Oficial da União, Brasília, DF, 3 out, 2017. Acesso em: 12 e agosto de 2018.

COELHO, G. M. P. et al. Educação permanente em saúde: experiência dos profissionais do serviço de atendimento móvel de urgência. Enferm. Foco, v. 4, n. 3,4, p. 161-163, 2013.

COHEN, J. Statistical power analysis. Curr Dir Psychol Sci, v. 1, n. 3, p. 98-101, 1992.

\section{DANTAS, R. A. N. Avaliação da qualidade da assistência prestada pelo Serviço de Atendimento}

Móvel de Urgência do Rio Grande do Norte. 2014. 139 f. Tese (Doutorado - Programa de PósGraduação em Ciências da Saúde, Centro de Ciências da Saúde, Universidade Federal do Rio Grande do Norte, Rio Grande do Norte, 2014.

DANTAS, R. A. N. et al. Instrumento para avaliação da qualidade da assistência pré-hospitalar móvel de urgência: validação de conteúdo. Rev Esc Enferm USP, v. 49, n. 3, p. 381-387, 2015.

GARÇON, T. L.; PUPULIM, J. S. L. Qualidade do atendimento pré-hospitalar móvel na perspectiva dos profissionais. Cienc Cuid Saude, v. 16, n. 4, p. 1-8, 2017.

MATA, K. S. S. et al. Entraves no atendimento pré-hospitalar do SAMU: percepção dos enfermeiros. Rev Enferm UFPE online, v. 12, n. 8, p. 2137-2145, 2018.

MELO, W. O. S. M. et al. Gestão da qualidade na saúde. Rev UNINGÁ, v. 18, n. 1, p. 24-28, 2014.

O’DWYER, G.; MATTOS, R. A. Cuidado integral e atenção às urgências: o serviço de atendimento móvel de urgência do Estado do Rio de Janeiro. Rev. Saúde Soc, v. 22, n. 1, p. 199-210, 2013.

O’DWYER, G. et al. O processo de implantação do Serviço de Atendimento Móvel de Urgência no Brasil: estratégias de ação e dimensões estruturais. Cad. Saúde Públ, v. 33, n. 7, e00043716, 2017.

OLIVEIRA, L. S. et al. Indicadores de qualidade nos Serviços de Urgência Hospitalar. Cad Grad Ciên Biol Saúde, v. 4, n. 3, p. 173-188, 2018.

ORTIGA, A. M. B. et al. Avaliação do Serviço de Atendimento Móvel de Urgência em Santa Catarina, Brasil. Cad. Saúde Públ, v. 32, n. 12, e00176714, 2016.

SERGIPE. Portal da Saúde. Sistema de dados e informações do samu revela números relacionados a 2017. Disponível em: http://saude.se.gov.br/index.php/2017/12/28/sistema-de-dados-einformacoes-do-samu-revela-numeros-relacionados-a-2017. Acesso em: 3 jun. 2018. 
SILVA, K. et al. Gestão da qualidade total nos serviços de saúde: modelo gerencial em desenvolvimento. Rev Eletr Gestão Saúde, v. 6, n. 1, p. 617-632, 2015.

SILVA, S. F. et al. Dificuldades vivenciadas em um serviço de atendimento móvel de urgência: Percepções da equipe de enfermagem. R. Enferm Cent $\mathbf{O}$ Min, v. 4, n. 2, p. 1161-1172, 2014.

VITURI, D. W. Acolhimento com classificação de risco em hospitais de ensino: avaliação da estrutura, processo e resultado. Rev. Latino-Am. Enferm, v. 21, n. 5, p. 1179-1187, 2013. 
1 Residente em Cuidado ao Paciente Crítico - Hospital Sírio Libanês; Graduado em Enfermagem.

E-mail: joathan.borges@souunit.com.br

2 Residente em Saúde do Adulto- Universidade Federal de Sergipe - UFS; Graduada em Enfermagem.

E-mail: jadiane_96@yahoo.com.br

3 Mestra em Saúde e Ambiente; Especialista em UTI e Emergência; Especialista em Enfermagem do Trabalho; Graduada em Enfermagem; Professora do Curso de Enfermagem, Universidade Tiradentes - UNIT.

E-mail: hendyaracarvalho@hotmail.com

4 Doutora em Biotecnologia Industrial - UNIT; Mestre em Ciências da Saúde - UFS; Especialista em Administração Hospitalar e em Educação Profissional na área da saúde; Professora do curso de Enfermagem - UNIT.

E-mail: danilima.lipe@gmail.com

5 Mestra em Enfermagem, Universidade Federal de Sergipe - UFS; Especialista em Urgência e Emergência, APH e Unidade de Terapia Intensiva; Professora do curso de Enfermagem - UNIT. E-mail: ingridenzo@yahoo.com.br

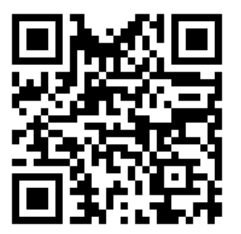

A autenticidade desse artigo pode ser conferida no site https://periodicos. set.edu.br

\section{(@) (1) ( )}

Este artigo é licenciado na modalidade acesso abertosob a Atribuição-Compartilhalgual CC BY-SA

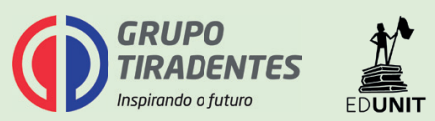

